\title{
Hydrodynamics of a Membrane Bound to a Substrate
}

\author{
Udo Seifert
}

Institut für Festkőrperforschung, Forschungszentrum Julich, D-52425 Jülich, Germany

Key Words: Membranes / Statistical Mechanics / Viscosity

\begin{abstract}
We briefly review recent theoretical work on the dynamical equilibrium shape fluctuations of a membrane interacting with a substrate. Solving the hydrodynamical equations we determine the damping rate $\gamma(q)$ as a function of the wave-vector $q$. For small $q$, we find universally $\gamma \sim q^{2}$. In an intermediate $q$-range, the damping rate behaves like either $\gamma \sim q^{6}$ (monotonic damping) or $\gamma \sim 1 / q$ (non-monotonic damping) depending on the relative sizes of the mean separation and the parallel correlation length, which are both determined by the adhesion potential. For large $q$, one recovers the behavior $\gamma \sim q^{3}$ of a free membrane. A numerical solution of the dispersion relation is presented for a membrane subject to electrostatic repulsion in a linear attractive potential.
\end{abstract}

\section{Introduction}

Membranes differ from liquid-gas interfaces in a fundamental aspect. Their conformation is not governed by surface tension but rather by bending energy [1]. Consequently, the dominant shape fluctuations are not capillary waves but bending excitations. The energy of such a bending mode is determined by the local curvature of the membrane and scales with the fourth power of the wave vector. Since the energy scale for these modes is comparable to the thermal energy, bending excitations are thermally excited. Due to the coupling of the membrane to the surrounding viscous fluid, these modes are overdamped. They can be observed in the microscope as pronounced flickering of giant vesicles $[2,3]$. In fact, a quantitative analysis of these modes for 
quasi-spherical vesicles yields a value for the bending rigidity $\kappa$ which is the essential material parameter for a membrane.

In this contribution, we briefly review recent theoretical work on the dynamics of an almost planar membrane in the vicinity of a substrate such as mica or glass $[4,5]$. From a practical point of view, orienting the membrane in a well defined geometry through the interaction with a substrate [6-9] should facilitate precise measurements since the slow translational and rotational motions of free vesicles are suppressed. More importantly, the shape fluctuations themselves are considerably affected by the presence of the substrate. Using a new technique, reflection interference microscopy [10-12], Rädler has demonstrated recently that dynamical fluctuations of a bound membrane can be characterized [13]. A quantitative comparison between experimental and theoretical work should then yield valuable information about the interaction of membranes with substrates.

\section{The Free Membrane}

It is instructive first to cast the seminal work of Brochard and Lennon [14] on the dynamics of a free membrane in a form which facilitates a qualitative understanding of the dynamics of a bound membrane. In the Monge representation, an almost planar membrane can be parametrized by $h(x, y)$. The bending energy stored in a displacement $h(x, y)=h_{q}\left(\mathrm{e}^{\mathrm{i} q x}+c c\right)$ described by a single Fourier-amplitude $h_{q}$ reads

$$
F=\frac{\kappa}{2} q^{4} h_{q} h_{q}^{*} \equiv \frac{1}{2} E(q) h_{q} h_{q}^{*},
$$

where $\kappa$ is the bending rigidity and $E(q)$ will be called the "energy" of this mode. Such a deformation leads to a restoring force $\delta F / \delta h_{q}^{*}$ which acts on the surrounding incompressible liquid. This liquid can be described within linearized hydrodynamics by the Stoke's approximation which reads $\nabla \cdot v=0$ and $\eta \nabla^{2} v=\nabla p$ for $z \neq 0$, where $z$ is the coordinate perpendicular to the membrane at $z=0$ and $\eta$ is the viscosity. At the membrane, the normal forces have to balance which implies $-\mathbf{T}_{z z}^{+}+\mathbf{T}_{z z}^{-}=-\delta F / \delta h_{q}$, where $\mathbf{T}_{z z}^{ \pm}$is the $(z, z)$-component of the liquid stress tensor $\mathbf{T}_{i j} \equiv$ $-p \delta_{i j}+\eta\left(\partial_{i} v_{j}+\theta_{j} v_{i}\right)$ at $z= \pm 0$.

These equations are solved with the following boundary conditions: (i) the velocity field in the liquid vanishes for $z \rightarrow \pm \infty$, (ii) continuity of the normal liquid velocity at $z=0$, (iii) impermeability of the membrane, i.e., $v_{z}(z= \pm 0)=\partial_{t} h_{q}$, (iv) continuity of the in-plane liquid velocity at $z=0$, (v) incompressibility of the membrane, which requires that the in-plane divergence of $v$ vanishes at $z=0$.

After eliminating the pressure and velocity field, one obtains an effective equation of motion for the membrane

$$
\partial_{1} h_{q}=-\Gamma(q) \delta F / \delta h_{q}^{*}=-\Gamma(q) E(q) h_{q} .
$$

Here $\Gamma(q)=1 / 4 \eta q$, which is basically the Fourier transformed of the Oseen tensor, can be interpreted as a kinetic coefficient which reflects the long-range character of the hydrodynamic damping. Solving the equation of motion yields $h_{\mathrm{q}}(t)=h_{q}(0) \mathrm{e}^{-\gamma(q) t}$ with the damping rate

$\gamma(q)=\Gamma(q) E(q)$.

Thus, $\gamma=\kappa q^{3} / 4 \eta$ for a free membrane. The form (3) of the damping rate as a product of a kinetic coefficient which contains the dissipation and an energy which contains the driving force will also persist for a bound membrane.

\section{The Bound Membrane}

A membrane interacts with a substrate through a potential $V(l)$ which contains the contributions from various forces such as electrostatic interactions, van der Waals forces, hydration and protrusion forces, and osmotic pressure. The energy $F$ of a membrane at $l(x, y)$ in such a potential $V(l)$ reads $[15,16]$

$$
F=\int \mathrm{d} x \mathrm{~d} y \frac{\kappa}{2}\left(\nabla^{2} l\right)^{2}+V(l) \approx \frac{\kappa}{2} \int \mathrm{d} x \mathrm{~d} y\left(\nabla^{2} h\right)^{2}+\xi^{-4} h^{2} .
$$

Here, we use a harmonic approximation for fluctuations $h(x, y) \equiv l(x, y)-l_{0}$ around the minimum of the potential at $l=l_{0}$ and define the parallel correlation length $\xi \equiv$ $\left(\kappa / \mathrm{d}^{2} V / \mathrm{d} l_{\mid l=l_{0}}^{2}\right)^{1 / 4}$. For a plane-wave bending mode with wave-vector $q$ and amplitude $h_{q}$,

$F \equiv \frac{1}{2} E(q, \xi) h_{q} h_{q}^{*}=\frac{\kappa}{2}\left(q^{4}+\xi^{-4}\right) h_{q} h_{q}^{*}$.

Thus, for small $q$, the energy $E(q, \xi)$ is dominated by the term $\xi^{-4}$ arising from the confining potential which is absent for a free membrane.

The equations for the surrounding liquid and the normal force balance are as in Sect. II, with $z=0$ replaced by $z=I_{0}$. While the boundary conditions (ii) through (v) still hold, the presence of the wall requires that the velocity field of the liquid vanishes at the substrate at $z=0$. Solving the hydrodynamic equations with a plane-wave dependency, one obtains a damping rate of the form [4]

$\gamma\left(q, l_{0}, \xi\right)=\Gamma\left(q, l_{0}\right) E(q, \xi)$

with the energy $E(q, \xi)$ given by (5) and the kinetic coefficient

$$
\begin{aligned}
& \Gamma\left(q, l_{0}\right) \equiv \\
& \frac{1}{2 \eta q} \frac{\sinh ^{2}\left(q l_{0}\right)-\left(q l_{0}\right)^{2}}{\sinh ^{2}\left(q l_{0}\right)-\left(q l_{0}\right)^{2}+\sinh \left(q l_{0}\right) \cosh \left(q l_{0}\right)+\left(q l_{0}\right)} \\
& \rightarrow \begin{cases}l_{0}^{3} q^{2} / 12 \eta, & q<1 / l_{0} \\
1 / 4 \eta q, & q>1 / l_{0}\end{cases}
\end{aligned}
$$


The asymptotic behavior of $\Gamma\left(q, I_{0}\right)$ can easily be understood as follows: For $q>1 / I_{0}$ the distortion of the velocity field in the liquid decays so fast that it does not feel the presence of the substrate. $\Gamma$ is then given by its form of a free membrane. For $q<1 / l_{0}$, the $q^{2}$-dependence arises from the fact that the volume of the liquid between the membrane and the substrate is conserved which leads to the usual quadratic dependence of the kinetic coefficient for conserved quantities.

Depending on the relative size of the crossover lengthscale $l_{0}$ of the kinetic coefficient and the crossover lengthscale $\xi$ of the energy, two different cases have to be distinguished for the $q$-dependency of the damping rate.

Monotonic damping: For $l_{0} \ll \xi$, one finds from $(6,7)$

$\gamma \approx \begin{cases}\kappa l_{0}^{3} q^{2} / 12 \eta \xi^{4}, & q \ll 1 / \xi \\ \kappa l_{0}^{3} q^{6} / 12 \eta, & 1 / \xi \ll q \ll 1 / l_{0} . \\ \kappa q^{3} / 4 \eta, & 1 / l_{0} \ll q\end{cases}$

For small $q$, the amplitude of the $q^{2}$ damping is thus determined by the potential through $\xi$ and $l_{0}$. The intermediate regime has been found previously in a related context by Brochard and Lennon [14]. They considered the fluctuations of two planar membranes separated by a distance $I_{0}$ as a crude model for a red-blood-cell. Since this distance was not enforced by a potential their treatment corresponds to $\xi=\infty$ for which the small $q$ regime is lost. For large $q$, one recovers, of course, the behavior of a free membrane.

Non-monotonic damping: For $\xi \ll l_{0}$, the $q$-dependency becomes

$$
\gamma \approx \begin{cases}\kappa l_{0}^{3} q^{2} / 12 \eta \xi^{4}, & q \ll 1 / l_{0} \\ \kappa / 4 \eta \xi^{4} q, & 1 / l_{0}<q<1 / \xi . \\ \kappa q^{3} / 4 \eta, & 1 / \xi \ll q\end{cases}
$$

The small and large $q$-behavior remains the same as above. However, in the intermediate range, the damping rate decreases with increasing wave-vector. As a numerical analysis of Eqs. (6) and (7) shows, the damping rate becomes non-monotonic whenever $\xi<0.24 I_{0}$.

Although the potential $V(l)$ determines both the lengths $I_{0}$ and $\xi$, whether it leads to monotonic or non-monotonic damping depends, in addition, on the strength of nonharmonic fluctuations, which can enhance the repulsion of the membrane from the substrate. According to Lipowsky's general classification [17] of adhesion potentials based merely on static properties, three regimes for the influence of fluctuations have to be distinguished: (i) The mean field regime, (ii) the weak fluctuation regime, and (iii) the strong fluctuation regime. Using an illustrative example, we now discuss the dynamical behavior in these three cases by relating them to the two different dynamical regimes described above.
The mean field regime: Consider a charged membrane pushed by an osmotic pressure $p$ towards a substrate. In weak electrolytes, where the screening length is large compared to $l_{0}$, fluctuations beyond the harmonic level can safely by ignored. The potential is then [16]

$V(l)=A / l+p l$,

where $A \equiv\left(\pi T / 2 l_{\mathrm{B}}\right)$. Here, $l_{\mathrm{B}} \simeq 0.7 \mathrm{~nm}$ is the Bjerrum length in water and $T$ the temperature (with Boltzmann's constant set to unity). One immediately gets $l_{0}=(A / p)^{1 / 2}$ and $\xi=(\kappa / 2 A)^{1 / 4} l_{0}^{3 / 4}$. In the small $q$ range, this implies that the damping rate $\gamma \approx(A / 6 \eta) q^{2}$ does not depend on the mean separation $l_{0}$ for this potential.

For a quantitative example, we show in Fig. 1 the dispersion relation derived for the potential (10) for three different values of the mean separation $I_{0}=4 \mathrm{~nm}, 40 \mathrm{~nm}$, and $1000 \mathrm{~nm}$. For the latter value, the damping rate becomes non-monotonic. In fact, as $p \rightarrow 0$, which corresponds to the unbinding transition $[15,18], l_{0}$ becomes much larger than $\xi$ and the intermediate range spreads. In practice, however, $l_{0}=1000 \mathrm{~nm}$ is a very large separation which would require an unrealistically weak screening.

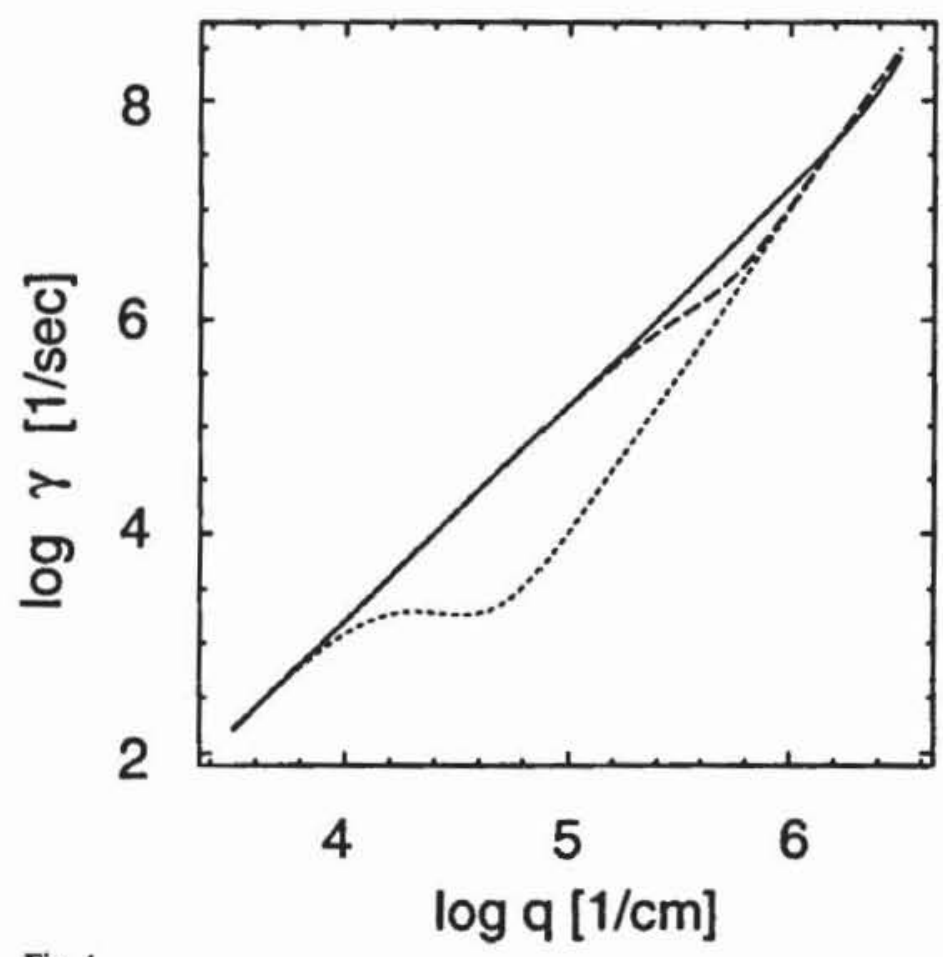

Fig. 1

Dispersion relation for a membrane subject to unscreened electrostatic interactions and an osmotic pressure (10). The separation $I_{0}$ is $4 \mathrm{~nm}$ (full line), $40 \mathrm{~nm}$ (dashed line) and $1000 \mathrm{~nm}$ (dotted line). The corresponding values of $\xi$ are $3.6 \mathrm{~nm}, 19 \mathrm{~nm}$, and $210 \mathrm{~nm}$, respectively. The bending rigidity is chosen as $\kappa=4 \times 10^{-13} \mathrm{erg}$, and $\eta=0.01 \mathrm{erg}$ $\mathrm{s} / \mathrm{cm}^{3}$ is the viscosity of water

The weak fluctuation regime: If the electrostatic repulsion is screened it becomes exponential in $l$. Nonharmonic fluctuations can then no longer be neglected. In a self consistent way they can be included by adding a steric interaction [6] $V_{\mathrm{FL}}=c\left(T^{2} / \kappa\right) / /^{2}$ to the effective potential, where 
$c$ is a numerical coefficient of order one. Ignoring the electrostatic repulsion for simplicity, the total potential now reads

$$
V(l)=c T^{2} / \kappa l^{2}+p l .
$$

The equilibrium separation is given by $l_{0}=(2 c / p)^{1 / 3} T^{2 / 3} / \kappa^{1 / 3}$ and the relation between the correlation length $\xi=(\kappa / T)^{1 / 2} I_{0} /(6 c)^{1 / 4} \simeq 3 l_{0}$ and $l_{0}$ becomes independent of the amplitude $p$ of the linear attractive potential. Since $\xi>I_{0}$, the weak fluctuation regime will always be governed by monotonic damping. Note that even for $p \rightarrow 0$ the intermediate behavior, $\gamma \sim q^{6}$, will be confined to the rather narrow interval $1 / 3 I_{0} \leqslant q \leqslant 1 / l_{0}$.

The strong fluctuation regime: If both the attractive as well as the repulsive potential become short ranged the nonharmonic fluctuations are so dominant that even the superposition of direct and steric potentials interaction fails to describe the interaction [15]. However, the scaling behavior of the damping rate given by (6) and (7) should still hold, provided one uses the fully renormalized $l_{0}$ and $\xi$.

\section{Dynamical Correlation Function}

Once the damping rate $\gamma\left(q, \xi, l_{0}\right)$ is determined, the dynamical correlation function for a bound membrane follows trivially as

$\left\langle h_{q}(t) h_{q}^{*}(0)\right\rangle=\frac{k_{\mathrm{B}} T}{E(q, \xi)} \exp \left[-\gamma\left(q, \xi, l_{0}\right) t\right]$.

\section{Possible Extensions}

The theory reviewed above can be extended in various directions. We close by mentioning two possible refinements. (i) A closer look on the dynamics of a free membrane, taking into account the bilayer character of a membrane, revealed that the dispersion relation deviates at small wavelength from the classical behavior due to the coupling of density and shape fluctuations [19]. This effect will persist for a bound membrane and has to be included in a quantitative comparison between theory and experiment. (ii) For most experiments, the bound membrane will be part of a giant vesicle adhering to the substrate. In this case, the closed topology imposes an additional constraint on the fluctuations. For a crude description of this effect is suffices to include a tension term $\Sigma q^{2}$ into the energy. The effect of such a term can then easily be worked out. However, if the wave length becomes comparable to the size of the bound part of the vesicle, the assumption of an infinite planar membrane breaks down. Since the dynamics then acquire a sensitive dependence on the shape of the bound vesicle, a theoretical treatment of this case will become highly non-trivial.

I thank the organizers for the opportunity to present this work at such an exciting meeting. Stimulating discussions with $\mathrm{M}$. Kraus, S. Langer, R. Lipowsky, J. Rådler, and E. Sackmann are gratefully acknowledged.

\section{References}

[1] For reviews on membranes, see, e.g. (a) R. Lipowsky, Nature 349, 475 (1991), and (b) R. Lipowsky, D. Richter, and K. Kremer (eds.) Structure and Conformation of Amphiphilic Membranes, Springer, Berlin 1992.

[2] H. Engelhardt, H.P. Duwe, and E. Sackmann, J. Phys. (France) Lettre 46, L395 (1985).

[3] I. Bivas, P. Hanusse, P. Bothorel, J. Lallane, and O. AguerreChariol, J. Phys. (France) 48, 855 (1987).

[4] U. Seifert, Dynamics of a bound membrane, Phys. Rev. E, in press.

[5] U. Seifert and S.A. Langer, Biophys. Chem., in press.

[6] W. Helfrich, Z. Naturforsch. 33a, 305 (1978).

[7] E. Evans, Colloids and Surfaces 43, 227 (1989).

[8] U. Seifert and R. Lipowsky, Phys. Rev. A 42, 4768 (1990).

[9] R. Lipowsky and U. Seifert, Langmuir 7, 1867 (1991).

[10] A. Zilker, H. Engelhardt, and E. Sackmann, J. Phys. (France) 48, 2139 (1987).

[11] A. Zilker, M. Ziegler, and E. Sackmann, Phys. Rev. A 46, 7998 (1992).

[12] J. Radler and E. Sackmann, Langmuir 8, 848 (1992).

[13] J. Radler, Thesis, TU-München (1993).

[14] F. Brochard and J.F. Lennon, J. Phys. (France) 36, 1035 (1975).

[15] R. Lipowsky and S. Leibler, Phys. Rev. Lett. 56, 2541 (1986).

[16] S. Leibler and R. Lipowsky, Phys. Rev. B35, 7004 (1987).

[17] R. Lipowsky, Phys. Scr. T29, 259 (1989).

[18] M. Mutz and W. Helfrich, Phys. Rev. Lett. 62, 2881 (1989).

[19] U. Seifert and S.A. Langer, Europhys. Lett. 23, 71 (1993).

Presented at the Discussion Meeting of the

E 8567 Deutsche Bunsen-Gesellschaft für Physikalische Chemie "Phase Transitions at Interfaces" in Bad Herrenalb, September 22nd to 24th, 1993 